Cancer/Radiother $1998: 2: 718-22$

(1) Elsevier, Paris

\title{
Les chimioradiothérapies dans les cancers du col utérin
}

\author{
P Maingon ', P Coucke ${ }^{2}$, C Haie-Méder ${ }^{3}$, I Barillot ${ }^{1}$ \\ ${ }^{1}$ Centre Georges-François-Lecierc, 1, rue du Professeur-Marion, 21034 Dijon: \\ ${ }^{3}$ institut Gustave-Roussy, 39, rue Camille-Desmoulins, 94805 Villejuif. France; \\ ${ }^{2}$ centre hospitalier universitaire Vaudois, $\mathrm{CH}-1011$ I ausanne, Suisse
}

\begin{abstract}
RÉSUMÉ
Le traitement des cancers du col utérin repose sur la radiothérapie et la chirurgie. Le pronostic des formes évoluées a conduit à proposer différentes modalités d'association dont seuls les traitements concomitants ont m̧ontré un intérêt. Les associations de radiothérapie et d'un radiosemsibilisant n'unt pas apporté de bénéfice, voire abouti à des résultats inférieurs à ceux de l'irradiation exclusive. L'hydroxyurée et la mitomycine $C$ ont été largement testées, seules ou en association, sans qu'elles puissent être retenues comme efficaces. Des associations modernes et des essais récents, il peut être établi que seu! le cisplatine (et peut-être ses dérivés) peut être retenu dans les propositions thérapeutiques futures. (C) 1998 Elsevier, Paris
\end{abstract}

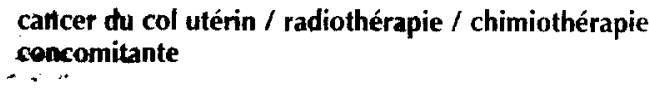

\section{SUAMARY}

\section{Concurrent chemoradiotherapy in cervix carcinomas.}

Treatment of uterine cervix carcinomas is based on radiotherapy and surgery. Prognosis of advanced carcinoma leads to the proposal of many combinations. Only concurrent radio-chemotherapy demonstrated some interests. Combination of radiotherapy and radiosensitisers failed to demonstrate any advantage and in some instances was associated with an adverse effect. Hydroxyurea and mitomycin $\mathrm{C}$ alone or associated were extensively tested without benefit. From modern combinations and recent studies, we could conclude that only cisplatin ind probably its derivates) can be included in future trials. (O) 1998 Flsevier, Paris

cervix carcinoma / radiotherapy / concurrent chemotherapy
Le traitement des cancers du col utérin repose sur la radiothérapie et la chirurgie. Dans les formes localisées, la radiothérapie exclusive [2] permet d'obtenir des résultats carcinologiques identiques à ceux de la combinaison radiothérapie-chirurgie [8]. Un essai randomisé a permis de confirmer l'équivalence de ces deux approches, en mettant toutefois en évidence une morbidité plus importante de l'association chirurgie-radiothérapie postopératoire [20]. Les auteurs recommandaient dans leur conclusion d'adapter les indications à chaque patiente.

Les principaux facteurs de pronostic défavorable sont constitués par l'atteinte ganglionnaire, le stade initial de la maladie et la taille de la tumeur. Le taux de survie spécifique à 5 ans des malades atteintes de cancer de stades I et II chute de 90 à $60 \%$ en présence d'un envahissement ganglionnairc pelvien $[2,8]$. Dans l'étude multifactorielle de 1875 patientes traitées par irradiation exclusive publiée par Barillot et al, il y avait une corrélation entre la survie spécifique et le contrôle local d'une part, et l'envahissement ganglionnaire et le stade clinique, d'autre part. La survie spécifique et la survie sans récidive étaient aussi significativement liées à la taille tumorale (supérieure à $5 \mathrm{~cm}$ ) [2]. Les ganglions pelviens et lomboaortiques constituent les sites principaux de récidive, ainsi que le centre du pelvis pour les tumeurs localement évoluées. Ces constatations ont été confirmées par l'analyse de 1499 patientes publiée par Perez et al [27]. L'irradiation prophylactique des territoires lomboaortiques n'améliore pas le pronostic des cancers de stade avancé [12]. L'essai de Rotman et al a cependant mis en évidence une diminution du taux d'évolution ganglionnaire et une augmentation de la survie dans une sélection de tumeurs localisées [30].

Il est ainsi possible de sélectionner les cancers dont le pronostic est indiscutablement défuvorable et pour lesquels l'hypothèse de l'amélioration des résultats par un traitement associé à l'irradiation doit être discutée. 
Toutes les modalités d'association de la radiothérapie et de la chimiothérapie ont été testées pour améliorer le pronostic des cancers à haut risque de rechute. La chimiothérapie néoadjuvante a un effet délétère sur la survie et est grevée d'une toxicité qui ne semble pas acceptable $[4,21$, $32,36]$. Les traitements adjuvants n'ont démontré aucun bénéfice. Les chimioradiothérapies concomitantes ont été les plus étudiées pour répondre à deux objectifs : sensibiliser les cellules à l'irradiation et éradiquer la maladie systémique microscopique. Les études préliminaires ont rapidement suggéré une amélioration du pronostic par cette approche. Les produits les plus communément utilisés sont la mitomycine C, l'hydroxyurée, le 5-fluoro-uracile et les sels de platine. D'autres facteurs potentialisant les effets de l'irradiation comme les radiosensibilisants ont largement été testés en clinique. Ils concernent des produits bioréductifs présentant une affinité sélective vis-àvis des cellules hypoxiques ou bien les associations chimioradiothérapie classiques.

\section{LES RADIOSENSIBILISANTS}

En marge des facteurs cliniques pronostiques, la recherche radiobiologique a permis d'identifier un certain nombre de facteurs de résistance aux traitements. L'hypoxie est considćrée comme une des causes principales de la résistance tumorale à l'irradiation, spécialement dans les cancers évolués du col utérin. I a $\mathrm{pO}_{2}$ intratumorale a été présentée comme un facteur pronostique de la survie [13]. Les travaux originaux de Bush et Overgaard et al ont permis d'établir la relation entre le taux d'hémoglobine et le contrôle local, sans lien avec la survenue d'échec à distance $[3,26]$. Ces données ont conduit à proposer l'utilisation des radiosensibilisants en association à la radiothérapie dans cette indication. Le premier d'entre eux, le misonodazole, a été introduit dés 1974 en association à l'irradiation externe [9]. Quatre essais ont été conduits avec cette molécule sans démontrer de bénéfice $[22,24,33,34]$. Des dérivés nitro-imidazolés (étanidazole et pimonidazole), moins neurotoxiques et plus efficaces in vitro, ont ensuite été testés. Le pimonidazole a fait l'objet d'une étude de phase III conduite par le Medical Research Council (MRC). L'association thérapeutique s'est montrée délétère sur le contrôle local et la survie, statistiquement meilleurs dans le bras radiothérapie seule [7]. L'apparition de nouveaux produits bioréductifs comme la tirazapamine va probablement relancer les études dans cette voie [18].

\section{La mitomycine $\mathrm{C}$}

L'association 5-fluoro-uracile--mitomycine $\mathrm{C}$ a surtout été étudiée par Thomas et al au Princess Margaret Hospital. Plus de 200 malades ont ainsi reçu une association de radiothérapie et de chimiothérapie selon ce protocole. Elle a permis d'obtenir une amélioration de $10 \%$ du taux de contrôle pelvien de la maladie dans une comparaison historique. Une analyse multifactorielle a surtout mis en évidence une augmentation très significative du taux de toxicité digestive avec cette association lorsqu'elle était comparée à l'utilisation du 5-fluoro-uracile seul (22\% versus $7 \%, p=0,005$ ) [38]. Par ailleurs, l'efficacité de cette association n'a pas été confirmée [5, 19].

\section{L'hydroxyurée}

Les études in vitro ont montré la sensibilisation des cellules tumorales par blocage dans une phase sensible du cycle cellulaire par l'hydroxyurée [31]. En 1970, un essai randomisé conduit par le Gynecologic Oncology Group (GOG) a comparé hydroxyurée $\left(80 \mathrm{mg} / \mathrm{m}^{2}\right.$ per os, tous les 3 jours pendant 12 semaines) à un placebo chez des patientes alleintes d'une tumeur de stade IIIB ou IVA. Les malades recevant l'hydroxyurée ont bénéficié d'un meilleur taux de réponse (68\% versus $48 \%$ ), d'un plus grand intervalle de survie sans progression (14 mois versus 8 mois) et d'une amélioration de la médiane de survie ( 20 mois versus 11 mois). Les critiques de cet essai sont nombreuses: staging initial non chirurgical, condition non optimale de la radiothérapie (dose limitée à 70 Gy en cas association radiothérapie externe-curiethérapie, $60 \mathrm{~Gy}$ en cas d'irradiation externe seule) et surtout plus de la moitié des malades inévaluables du fait d'une durée de surveillance insuffisante [14].

Le second essai randomisé du GOG a pris une toute autre direction. La sélection des tumeurs en fonction du stade clinique était identique mais le staging comportait un curage lomboaortique. L'essai comparait une radiotherapie exclusive à une irradiation associée à une immunothérapie par le Corynébactérium parvum. Aucune différence n'a été montrée entre les deux bras de traitement [6]. Cette étude a surtout permis de confirmer le pronostic défavorable des tumeurs associées à un envahissement ganglionnaire lomboaortique, et ces malades atteintes de métastases lomboaortiques ont été exclues des essais ultérieurs par la réalisation systématique d'un curage ganglionnaire lomboaortique lors du bilan initial. Néanmoins, l'association chimioradiothérapie allait être considérée comme le traitement de référence pour ces malades.

Le troisième essai du GOG a comparé deux modalités d'association chimioradiothérapie : irradiation plus hydroxyurée versus irradiation plus misonidazole [34]. L'analyse de l'essai qui a inclus 294 malades a permis de conclure à une supériorité du bras hydroxyurée en termes de survie sans progression $(p=0,05)$ et de survie globale $(p=0,07)$. Le pronostic des cancers de stade IIB s'est révélé identique dans les deux bras et comparable à celui des tumeurs de stades IIIB et IVA traités par irradiation et hydroxyurée. Le pronostic des cancers de stade IIIB et IVA traités par l'association radiothérapie-misonidazole 
s'est avéré défavorable. L'interprétation de cet essai n'est pas univoque. L'efficacité de l'hydroxyurée comme radiosensibilisant peut être discutée si on n'exclut pas l'hypothèse selon laquelle l'utilisation des dérivés nitroimidazolés peut conduire à une diminution du taux de contrôle pelvien $[7,22]$.

\section{Le cisplatine et ses associations}

Les étapes suivantes ont été marquées par la mise au point de la radiochimiothérapie concomitante «standard»: 1'association de radiothérapie, 5-fluoro-uracile et de cisplatine. La combinaison du 5-fluoro-uracile et du cisplatine a fait l'objet de nombreuses études dont la caractéristique commune était d'inclure moins de 40 malades. La conclusion qui peut en être rapportée concerne la tolérance de l'association qui semble acceptable.

Le GOG a entrepris une comparaison entre l'hydroxyurée et une association de 5-fluoro-uracile et de cisplatine. Soixante-quinze patientes ont été traitées dans un essai de phase I-II testant l'association des trois agents et de la radiothérapie [35]. L'hydroxyurée était délivrée per os, deux fois par semaine, à la dose de $2,5 \mathrm{~g} / \mathrm{m}^{2}$, le cisplatine à j1 et $\mathrm{j} 29$ à la dose de $50 \mathrm{mg} / \mathrm{m}^{2}$ et le 5-fluoro-uracile en perfusion de 96 heures à la dose initialc de $800 \mathrm{mg} / \mathrm{m}^{\text {? }}$ de j2 à j5 ct de j30 à j33. Lc taux de réponse (complète et partielle) était de $93 \%$. Une toxicité de grade 3 ou 4 a été observée chez $55 \%$ des malades. Les auteurs ont alors estimé que ce schéma pouvait être évalué dans un essai de phase III en réduisant la dose d'hydroxyurée. L'essai de phase III dont les résultats préliminaires ont été présentés par Rose et al au congrès de l'American Society of Clinical Oncology (ASCO) de 1998 permet d'apporter des réponses à différentes questions [29]. Cinq cent soixante-quinze malades ont été traitées par une association chimioradiothérapie, la radiothérapie étant identique dans les trois bras de l'étude et délivrait $80 \mathrm{~Gy}$. La randomisation portait sur la chimiothérapie: les patientes incluses dans le bras A recevaient du cisplatine $\left(40 \mathrm{mg} / \mathrm{m}^{2}\right.$ ) chaque semaine; dans le bras $\mathbf{B}$, elles recevaient une association de cisplatinc $\left(50 \mathrm{mg} / \mathrm{m}^{2}\right.$, jl et j29), de 5-fluoro-uracile ( $1 \mathrm{~g} \cdot \mathrm{m}^{-2}, \mathrm{j}^{-1}$ en perfusion de 96 heures, j1 et j29) et d'hydroxyurée ( $2 \mathrm{~g} / \mathrm{m}^{2}$, deux fois par semaine) et dans le bras $C$ de l'hydroxyurée seule ( $3 \mathrm{~g} / \mathrm{m}^{2}$, deux fois par semaine). Il existait une amélioration significative de la survie sans progression et de la survie globale dans les bras A et B par rapport au bras $C$ (sans cisplatine). La tolérance immédiate était nettement meilleure dans le bras cisplatine hebdomadaire. Il faut être prudent dans l'interprétation de ces résultats, surtout en ce qui concerne la toxicité tar dive, étant donné que le recul n'était que de 24 mois. On peut retenir la supériorité du sel de platine sur l'hydroxyurée, l'absence d'effet additif avec l'association des deux agents et l'absence de supériorité de l'associa- tion 5-fluoro-uracile-cisplatine sur le platine seul. La publication récente d'un essai randomisé mené à Toronto confirme l'absence de bénéfice apporté par l'addition de 5-fluoro-uracile à la radiothérapie [37]. Il faut toutefois observer que le protocole d'administration de la chimiothérapie était vraisemblablement non optimal (5-fluorouracile, $1 \mathrm{~g} / \mathrm{m}^{2}, \mathrm{j} 1$ à $\mathrm{j} 4$ et les 4 derniers jours de l'irradiation externe).

L'association 5-fluoro-uracile-mitomycine C-cisplatine a été proposée en 1983 dans une étude pilote menée à l'Universty of California - San Francisco (UCSF) sur dix patients [15]. Le Radiation Therapy Oncology Group (RTOG, protocole 85-15) et le North Central Oncology Group (NCOG) ont mené une étude similaire sur 38 malades. Les patientes recevaient une radiothérapie externe de 45 Gy et une curiethérapie en deux temps à bas débit de dose. La chimiothérapie consistait en une association de 5 -fluoro-uracile $\left(1 \mathrm{~g} / \mathrm{m}^{2}, \mathrm{j} 1\right.$-j4 puis lors de la 1 re application de curiethérapie), mitomycine $C$ $\left(10 \mathrm{mg} / \mathrm{m}^{2}\right.$ par voie intraveineuse en bolus, $\mathrm{jl}$ ) et cisplatine $\left(75 \mathrm{mg} / \mathrm{m}^{2}, j 1\right.$ de la seconde injection de 5 -fluorouracile). Le taux de réponse complète et de survie à 2 ans était de $63 \%$ [17]. Une étude de seconde génération, basée sur la même association mais avec une réduction de $25 \%$ de la dose de mitomycine $\mathrm{C}$ et une augmentation de $10 \%$ de la dose délivréc par radiothérapic aux paramètres, a permis d'obtenir une diminution du taux de toxicité hémato-logique de grade $4(2 \%)$ et non hématologique de grade $3(8 \%)$. Le taux de réponse complète était en revanche augmenté de 63 à $77 \%$. Le taux survie à 1 an était également meilleur ( $81 \%$ versus $71 \%$ ) [16]. Il faut en retenir l'importance considérable de la qualité de la radiothérapie. L'apport de la dosimétrie moderne pour la contribution de la curiethérapie permet en effet de diminuer le taux des complications en améliorant sensiblement le pronostic de ces cancers.

L'utilisation des sels de platine peut s'étendre avec l'introduction du carboplatine comme agent radiosensibilisant. Une étude de phase I-II vient de confirmer la bonne tolérance immédiate d'un traitement associant une radiothérapie externe de 46 Gy complétée par une application de curiethérapie à haut débit de dose délivrant 8 Gy au point $\mathrm{A}$ et une chimiothérapie par le carboplatine perfusion continue $\left(12 \mathrm{mg} \cdot \mathrm{m}^{-2} \cdot \mathrm{j}^{-1}\right)$ [23]. L'étude pharmacocinétique associée a montré qu'à cette dose, la concentration en sels de platine dans le plasma et dans la tumeur restait inférieure à celle nécessaire à l'obtention d'une radiopotentialisation in vitro. D'autres études sont nécessaires pour évaluer cette association [25]. L'association de carboplatine et 5-fluoro-uracile a été testée chez 74 patientes traitées par irradiation précédant une chirurgie d'exérèse. L'analyse des résultats a confirmé la bonne tolérance du protocole. La comparaison de ces résultats avec ceux d'une série historique, plaidaient en faveur de la poursuite des études avec la molécule [28]. 
Il n'en reste pas moins que l'association chimioradiothérapie n'est toujours pas considérée par de grands spécialistes de la gynécologie tumorale comme le traitement de référence des cancers localement évolués du col utérin à la fois en raison d'une toxicité jugée rédhibitoire et d'une efficacité incertaine $[10,11]$. À l'appui de cette thèse, deux essais randomisés ont comparé radiothérapie seule et association chimioradiothérapie. Le premier publié par Wong et al, dans lequel le cisplatine était délivré une fois par semaine dans un groupe et deux fois par semaine dans un autre groupe avec cisplatine, n'a pas montré de différence avec le bras contrôle radiothérapie seule [40]. Le deuxième essai, plus récent, associait pour quatre cures, dans le bras chimioradiothérapie, le cisplatine $\left(50 \mathrm{mg} / \mathrm{m}^{2}\right.$ à $\left.\mathrm{j} 1\right)$, la vincristine $\left(1 \mathrm{mg} / \mathrm{m}^{2}\right.$ à $\left.\mathrm{j} 2\right)$ et la bléomycine $\left(25 \mathrm{mg} / \mathrm{m}^{2}\right.$ de $\mathrm{j} 2$ à j4), délivrés toutes les 3 semaines. Le taux de réponse était de $88 \%$ dans le bras chimioradiothérapie contre $74 \%$ pour le bras radiothérapie seule $(p=0,04)$. La toxicité précoce apparaissait plus importante dans le bras chimioradiothérapie sans différence statistique à long terme ( $23 \%$ versus $13 \%)$. Avec une durée médiane de surveillance de 47 mois, les taux de survie sans récidive ( $52 \%$ versus $53 \%$ ) et de survie globale (62\% versus $64 \%$ ) à 3 ans n'étaient pas différents [39].

\section{CONCLUSION}

L'association chimioradiothérapie concomitante est une des voies les plus prometteuses pour l'amélioration du pronostic des cancers localement évolués du col utérin. De nombreuses questions restent en suspens. Le schéma optimal d'association doit être défini dans le cadre d'études contrôlées. Si le cisplatine s'impose comme un produit essenticl dans cette indication, son mode d'administration (bolus ou perfusion quotidienne ou injection hebdomadaire) reste sujet à discussions. Ses dérivés méritent des études complémentaires avant d'être évalués en pratique courante. Son association aux nouveaux produits bioréductifs constitue une voie de recherche parmi les plus intéressantes [1].

\section{RÉFÉRENCES}

I Aghajanian C, Brown C, O’Flaferty C, Fleischauer A, Curtin J, von Roemeling $\mathrm{R}$ et al. Phase I study of tirapazamine and cisplatin in patients with recurrent cervical cancer. Gynecol Oncol 1997; 67 : $127-37$

2 Barillot I, Horiot JC, Pigneux J, Schraub S, Pourquier H, Daly N et al. Carcinoma of the intact uterine cervix treated with radiotherapy alone. Int J Radiat Oncol Biol Phys $1997 ; 38$ : 969-78

3 Bush RS. The significance of anemia in clinical radiation therapy. Int J Radiat Oncol Biol Phys $1986 ; 12: 2047-50$

4 Chauvergne J, Lhommé C, Rohart J, Héron JF, Ayme Y, Goupil A ct al. Chimiothérapic néoadjuvante des cancers du col utérin aux stades Ilb et IIlb. Résultats éloignés d'un essai randomisé pluricentrique portant sur 151 patients. Bull Cancer $1993 ; 80: 1069-79$
5 Christie DR, Bull CA, Gebski V, Langlands A. Concurrent 5-fluorouracil, mitomycin $C$ and irradiation in locally advanced cervix cancer. Radiother Oncol $1995 ; 37: 181-9$

6 Disaia PJ, Bundy BN, Curry SL, Schlaerth J, Thigpen JT. Phase III study of the treatment of women with cervical cancer stage IIB, IIIB and IVA (confined to the pelvis and/or in paraaortic nodes) with radiotherapy alone versus radiotherapy plus immunotherapy with intravenous corynebacterium parvum): a GOG study. Gynecol Oncol $1987 ; 26: 386-94$

7 Dische S, Chassagne D, Hope-stone HF, Dawes PJDK, Roberts JT, Yosef $\mathrm{H}$ et al. A trial of Ro 03-8799 (pimonidazole) in carcinoma of the uterine cervix: an interim report of the Medical Research Council Working Party on Advanced Carcinoma of the Cervix. Radiother Oncol $1993 ; 26: 93-103$

8 Gerbaulet A, Kunkler IH, Kerr GR, Haie C, Michel G, Prade M et al. Combined radiotherapy and surgery: local control and complications in early carcinoma of the uterine cervix. The Villejuif experience 1975-1984. Radiother Oncol 1992; 23:66-73

9 Gray AJ, Dische S, Adams GE, Flockart IR, Foster JL. Clinical testing of the radiosensitizer Ro 03-0582. I. Dose tolerance and tumor concentration. Clin Radiol 1976;27:151-7

10 Grigsby PW, Graham MV, Perez CA, Galakatos AE, Camel HM, Kan MS. Prospective phase $\mathrm{I} / \mathrm{II}$ studies of definitive irradiation and chemotherapy for advanced gynecologic malignancies. Am J Clin Oncol 1996;19:1-6

11 Grigsby PW, Lu JD, Mutch DG, Kim RY, Eifel P. Twice daily fractionation of external irradiation with brachytherapy and chemotherapy in carcinoma of the cervix with positive para-aortic lymph nodes: a phase II study of the Radiation Therapy Oncology Group 92-10. Int J Radiat Oncol Biol Phys $1998 ; 41$ : $817-22$

12 Haie C, Pejovic MH, Gerbaulet A, Horiot JC, Pourquier H, Delouche $J$. Is prophylactic paraaortic irradiation worthwhile in the treatment of advanced cervical carcinoma? Results of a controlled clinical trial of the EORTC radiotherapy group. Radiother Oncol $1988 ; 11: 101-12$

13 Höckel M, Schlenger K, Aral B, Mitze M, Schaffer U, Vaupel P. Association between tumor hypoxia and malignant progression in advanced cancer of the uterine cervix. Cancer Res 1996; 56 : 4509-15

14 Hreschchnyshyn MM, Aron BS, Boronow RC, Franklin E, Shingleton HM, Blessing JA. Hydroxyurea versus placebo combined with radiation to treat stages IIIB or IVA cervical cancer confined to the pelvis. Int J Radiat Oncol Biol Phys $1979 ; 5: 317-26$

15 John M, Cooke K, Flam M, Padmanabhan A, Mowry PA. Premliminary results of concomitant radiotherapy and chemotherapy in advanced cervical cancer. Gynecol Oncol $1987 ; 28: 101-5$

16 John M, Flam M, Markus M. A second generation phase II chemoradiation protocol for advanced cervical cancer. Proc Am Soc Clin Oncol 1991:10:190

17 John M, Flam M, Sikic B, Rotman Cooper J, Malec M. Preliminary results of concurrent radiotherapy and chemotherapy in advanced cervical carcinoma: a phase I-II prospective intergroup NCOGRTOG study. Gynecol Oncol $1990 ; 37: 1-5$

18 Kapp DS, Giaccia AJ. New directions for biology research in cancer of the uterine cervix. JNCI Monographs $1996 ; 21: 131-9$

19 Khoury GG, Bulman AS, Joslin CA. Rotwell RI. Concomitant pelvic irradiation, 5-fluorouracil and mitomycin $C$ in the treatment of advanced cervical carcinoma. Br J Radiol 1991: 64 : $252-60$

20 Landoni F, Maneo A, Colombo A, Placa F, Milani R, Perego P et al. Randomized study of radical surgery versus radiotherapy for stage I-II cervical cancer. Lancet $1997 ; 350: 535-40$

21 Leborgne F, Leborgne JH, Doldan R, Zubizaretta E, Ortega B, Maisonneuve $J$ et al. Induction chemotherapy and radiotherapy of advanced cancer of the cervix: a pilot study and a phase III randomized trial. Int J Radiat Oncol Biol phys $1997 ; 10: 343-50$ 
22 Leibel S, Baucr M, Wasserman T, Marcial V, Rotman M, Horn back $\mathrm{N}$ et al. Radiotherapy with or without misonidazole for patients with stage IIIB or IVA of the uterine cervix. Int J Radiat Oncol Biol Phys $1987 ; 13: 541-9$

23 Micheletti E, La Face B, Bianchi E et al. Continous infusion of carboplatin during conventional radiotherapy treatment in advanced squamous carcinoma of the uterine cervix IIB-IIIB (UICC). A phase I/II and pharmacokinetic study. Am J Clin Oncol 1997; 20:613-20

24 Medical Research Council trial of misonidazole in carcinoma of the uterine cervix. A report from the MRC Working party on misonidazole for cancer of the cervix. Br J Radiol $1984 ; 57: 491-9$

25 Muderspach LI, Curtin JP, Roman LD, Gebhardt JA, Klement V, Qian D et al. Carboplatin as a radiosensitizer in locally advanced cervical cancer: a pilot study. Gynecol Oncol $1997 ; 65: 336-42$

26 Overgaard J, Bentzen SM, Kolstad P, Kjoerstad K, Davy M, Bertelsen $\mathrm{K}$ et al. Misonidazole combined with radiotherapy in the treatment of carcinoma of the uterine cervix. Int $J$ Radiat Oncol Biol Phys 1989; $16: 1069-72$

27 Perez CA, Grigsby PW, Chao KSC, Mutch DG, Lockett MA. Tumor size, irradiation dose and long term outcome of carcinoma of uterine cervix. Int J Radiat Oncol Biol Phys 1998;41:307-17

28 Pras E, Willemse PH, Boonstra H, Hollema H, Heester MA, Szabo BG et al. Concurrent chemo-and radiotherapy in patients with locally advanced carcinoma of the cervix. Ann Oncol 1996; 7:511-6

29 Rose B, Bundy B, Thigpen J. Preliminary results of a phase III randomized study of concomitant chemotherapy with weekly cisplatin versus hydroxyurea, 5-FU infusion and bolus cisplatin versus hydoxyurea in advanced cervical cancer: a GOG study [abstract]. Proceeding ASCO 1998

30 Rotman M, Choi K, Guze C, Marcial V. Hornback N, John M. Prophylactic irradiation of the paraaortic lymph node chain in stage IIB and bulky stage I carcinoma of the cervix. Int $J$ Radiat Oncol Biol Phys 1990; 19 : 513-21

31 Sinclair $W$. The combined effect of hydroxyurea and $X$-rays on Chinese hamster cells in vitro. Cancer Res $1968 ; 52: 601-5$
32 Souhami L, Gil R, Allan SE, Canary VPC, Araujo CMMM, Pinto LH et al. A randomized trial of chemotherapy followed by pelvic radiation therapy in stage IIIB carcinoma of the cervix. $J$ Clin Oncol $1991 ; 9: 970-7$

33 Stehman FB, Bundy BN, Thomas G, Keys HM, d'Ablaing G, Fowler $W C$ et al. Hydroxyurea versus misonidazole with radiation in cervical carcinoma: long term follow-up of a Gynecology Oncology Group. J Clin Oncol 1993; 11 : 1523-8

34 Stehman FB, Bundy BN, Keys H, Currie JL, Mortel R, Creasman WT A randomized trial of hydroxyurea versus misonidazole adjunct to radiation therapy in carcinoma of the cervix: a preliminary report of a Gynecology Oncology Group Study. Am J Obstet Gynecol $1988 ; 159: 87-94$

35 Stehman FB, Bundy BN, Kucera PR, Deppe G, Reddy S, O'Connor DM. Hydroxyurea, 5-fluorouracil infusion and cisplatin adjunct to radiation therapy in cervical carcinoma: a phase I-II trial of the Gynecology Oncology group. Gynecol Oncol $1997 ; 66: 262-7$

36 Tattersall, MHN, Ramirez C, Coppleson M. A randomized trial comparing platinum-based chemotherapy followed by radiotrherapy alone in patients with locally advanced cervical cancer. Int $J$ Gynecol Oncol $1992 ; 2: 224-51$

37 Thomas G, Dembo A, Ackerman I, Franssen E, Balogh J, Fyles A et al. A randomized trial of standard versus partially hyperfractionated radiation with or without concurrent 5-fluorouracil in locally advanced cervical cancer. Gynecol Oncol $1998 ; 69: 137-45$

38 Thomas G, Dembo A, Fyles F, Gadalla T, Bcalc F, Bcan H et al, Concurrent chemoradiation in advanced cervical cancer. Gynecol Oncol $1990 ; 38: 446-52$

39 Tseng CJ, Chang CT, Lai CH, Soong YK, Hong JH, Tang SG et al. A randomized trial of concurrent chemoradiotherapy versus radiotherapy in advanced carcinoma of the uterine cervix. Gynecol Oncol $1997 ; 66: 52-8$

40 Wong LC, Choo YC, Choy D, Sham JS, Ma HK. Long term follow-up of potentiation of radiotherapy by cisplatinum in advanced cervical cancer. Gynecol Oncol 1989 : 35 : 159-63 